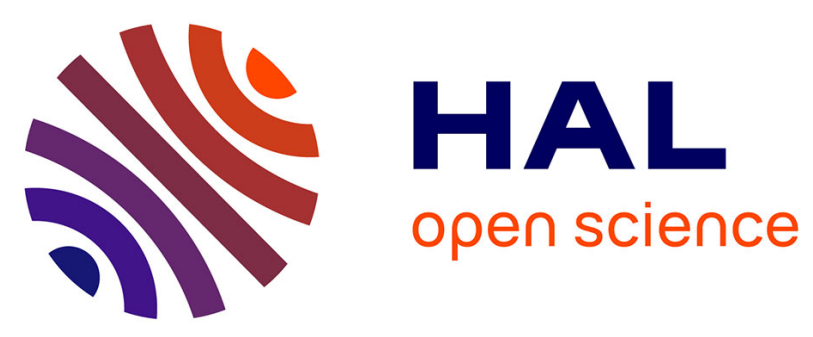

\title{
Oxidation-Induced Surface Roughening of Aluminum Nanoparticles Formed in an Ablation Plume
}

Georg Daniel Förster, Marie Girault, Jérôme Menneveux, Luc Lavisse, Jean-Marie Jouvard, Maria del Carmen Marco de Lucas, Valérie Potin, François-Xavier Ouf, Moussa Kerkar, Jean-Luc Le Garrec, et al.

\section{To cite this version:}

Georg Daniel Förster, Marie Girault, Jérôme Menneveux, Luc Lavisse, Jean-Marie Jouvard, et al.. Oxidation-Induced Surface Roughening of Aluminum Nanoparticles Formed in an Ablation Plume. Physical Review Letters, 2015, 115 (24), pp.246101. 10.1103/PhysRevLett.115.246101 . hal01244599

\author{
HAL Id: hal-01244599 \\ https://hal.science/hal-01244599
}

Submitted on 11 Feb 2021

HAL is a multi-disciplinary open access archive for the deposit and dissemination of scientific research documents, whether they are published or not. The documents may come from teaching and research institutions in France or abroad, or from public or private research centers.
L'archive ouverte pluridisciplinaire HAL, est destinée au dépôt et à la diffusion de documents scientifiques de niveau recherche, publiés ou non, émanant des établissements d'enseignement et de recherche français ou étrangers, des laboratoires publics ou privés. 


\title{
Oxidation-Induced Surface Roughening of Aluminum Nanoparticles Formed in an Ablation Plume
}

\author{
Georg Daniel Förster, ${ }^{1}$ Marie Girault, ${ }^{2}$ Jérôme Menneveux, ${ }^{1}$ Luc Lavisse, ${ }^{2}$ Jean-Marie Jouvard, ${ }^{2}$ \\ Maria del Carmen Marco de Lucas, ${ }^{2}$ Valérie Potin, ${ }^{2}$ François-Xavier Ouf, ${ }^{4}$ Moussa Kerkar, ${ }^{5}$ Jean-Luc Le Garrec, \\ Erwann Carvou, ${ }^{7}$ Sophie Carles, ${ }^{7}$ Franck Rabilloud, ${ }^{1}$ Florent Calvo, ${ }^{3}$ Jin Yu, ${ }^{1,6, *}$ and James Brian Mitchell ${ }^{7, \dagger}$ \\ ${ }^{1}$ Institut Lumière Matière, UMR5306 Université Lyon 1-CNRS, Université de Lyon, 69622 Villeurbanne Cedex, France \\ ${ }^{2}$ Laboratoire Interdisciplinaire Carnot de Bourgogne, UMR 6303 CNRS-Université Bourgogne Franche-Comté, \\ 9 Av. A. Savary, BP 47870, 21078 Dijon Cedex, France \\ ${ }^{3}$ Laboratoire Interdisciplinaire de Physique, CNRS-Université Joseph Fourier, \\ UMR 5588, BP 87, 38402 Saint Martin d'Hères, France \\ ${ }^{4}$ Institut de Radioprotection et de Sûreté Nucléaire (IRSN), PSN-RES, SCA, LPMA, Gif sur Yvette 91192, France \\ ${ }^{5}$ Laboratoire de Mécanique, Matériaux et Énergétique, Faculté des Sciences Exactes, \\ Université A. MIRA de Béjaïa, Route de Targa Ouzemour, 06000 Béjaïa, Algeria \\ ${ }^{6}$ Key Laboratory for Laser Plasmas (Ministry of Education), Department of Physics and Astronomy, \\ Shanghai Jiao Tong University, Shanghai 200240, China \\ ${ }^{7}$ Institut de Physique de Rennes, UMR 6251 CNRS-Université de Rennes 1, 35042 Rennes Cedex, France \\ (Received 11 July 2015; revised manuscript received 6 October 2015; published 11 December 2015)
}

\begin{abstract}
Nanoparticles formed within an ablation plume produced by the impact of a nanosecond laser pulse on the surface of an aluminum target have been directly measured using small-angle $\mathrm{x}$-ray scattering. The target was immersed in an oxygen-nitrogen gas mixture at atmospheric pressure with the $\mathrm{O}_{2} / \mathrm{N}_{2}$ ratio being precisely controlled. The results for an increasing oxygen content reveal remarkable effects on the morphology of the generated particles, which include a decrease in the particle volume but a marked increase in its surface ruggedness. Molecular dynamics simulations using a reactive potential and performed under similar conditions as the experiment reproduce the experimental trends and show in detail how the shape and surface structure of the nanoparticles evolve with increasing oxygen content. This good agreement between in situ observations in the plume and atomistic simulations emphasizes the key role of chemical reactivity together with thermodynamic conditions on the morphology of the particles thus produced.
\end{abstract}

DOI: 10.1103/PhysRevLett.115.246101

PACS numbers: 81.15.Fg, 31.15.xv, 52.38.Mf, 82.33.Xj

Laser ablation is the basis of key technologies such as pulsed laser deposition (PLD) for the production of nanostructures or thin films [1,2]. Metallic oxides, in particular, have received significant attention owing to promising optoelectronic applications with prominent examples being $\mathrm{Y}-\mathrm{Ba}-\mathrm{Cu}$ oxide for high-temperature superconductor thin films [3], or $\mathrm{ZnO}$ transparent conducting oxide for displays and solar cells [4]. The surface morphology and chemical composition of the thin films are determined by the properties of the oxide nanoparticles formed in the plume before their deposition onto the substrate [5]. However, the physical and chemical processes involved in particle formation in laser ablation plumes are numerous and complex and, especially in the case of metal oxides, not understood in great detail. In situ observation of oxide nanoparticles formed within an ablation plume is required, therefore, primarily to understand the mechanisms involved in thin film formation, but equally importantly, to provide experimental control of the thin film properties through experimental conditions. Ultraviolet Rayleigh scattering (RS) has been used to visualize a cloud of gas-suspended nanoparticles in the range of typically several $\mathrm{nm}$ [6]. Photoluminescent (PL) nanoparticles can be detected efficiently from their emission spectra [7]. Time-resolved images of nanoparticles produced in an ablation plume have been obtained in situ using RS and PL techniques [8]. However, the information provided by these optical diagnostics is limited to the macroscopic hydrodynamic properties of the particles, including their kinetic energy and the morphology of the particle cloud. Although the global thermodynamic properties in an ablation plume are reasonably well understood owing to dedicated modeling [9-11], the influence of a reactive ambient gas on individual particles, and, in particular, on their structure at the microscopic level, has remained undocumented so far.

In this Letter, we report observations on the influence of oxidation on the morphology of nanoparticles formed within an ablation plume produced by infrared nanosecond laser pulses impacting on a pure aluminum target immersed in a reactive oxygen-nitrogen gas mixture at atmospheric pressure. The small-angle x-ray scattering (SAXS) technique was used to enable in situ observations within the plume [12]. To the best of our knowledge, the influence of the oxygen content in the ambient gas on the morphological parameters of free particles in an ablation plume could be observed for the first time. The experimental results are interpreted using dedicated simulations performed under 
similar conditions and based on molecular dynamics with a reactive potential. The good agreement between the experiment and the simulation emphasizes the importance of chemical and, especially, oxidation reactions on the morphology of the generated nanoparticles. The success of the present approach establishes molecular simulation as a powerful tool for describing nucleation processes within an ablation plume, as a complementary method to traditional theoretical approaches based on classical or coarse-grained descriptions such as hydrodynamics [9], gas dynamics [10], or mesoscale Monte Carlo simulations [11].

The experiment was performed at the SOLEIL synchrotron facility in Saclay, France. Pulses from a Nd:YAG laser at $1064 \mathrm{~nm}$ with a pulse energy of $0.57 \mathrm{~mJ}$, a pulse duration of $5 \mathrm{~ns}$, and a repetition rate of $20 \mathrm{kHz}$ were focused on the sample surface on a spot of $110 \mu \mathrm{m}$ in diameter, resulting in an irradiance of $1.2 \mathrm{GW} / \mathrm{cm}^{2}$ impacting the aluminum target [Fig. 1(a)]. A permanent cloud of nanoparticles was formed above the target with a hemispherical form of several $\mathrm{mm}$ in diameter, as reported in a previous work [13]. An oxygen-nitrogen gas mixture was passed through the reaction chamber containing the target plate, in a direction perpendicular to the ablation laser beam with a flow rate of 1 liter per minute and an oxygen volume content $c_{\mathrm{O}}$ varying from $0 \%$ to $20 \%$ adjusted by mass flow controllers. Along the axis perpendicular to the ablation laser beam and the gas flow, an x-ray beam [see Fig. 1(a)] delivered by the SWING beam line [14], entered and exited the ablation chamber through x-ray transparent, air-tight mica windows. The $\mathrm{MHz}$ repetition rate of the SWING beam line allowed the $\mathrm{x}$-ray beam to be considered as continuous. The beam, with a cross section of $80 \times 300 \mu \mathrm{m}^{2}$ (height $\times$ width), passed through the middle of the ablation plume. The height of the $\mathrm{x}$-ray beam was set to $0.8 \mathrm{~mm}$ above the target surface, which was found with a vertical profile of the plume to optimize the scattering signal. For this $\mathrm{x}$-ray beam position, we expect a uniform mixing between the aluminum vapor and the ambient gas resulting from the mutual diffusion of the two species across their interface. An x-ray energy of $12 \mathrm{keV}$, corresponding to a wavelength of $1 \AA$, was used for these measurements. The distance between the ablation plume and the detector was set to 4 meters and the $q$ range covered in this configuration was 0.0022 to $0.2 \AA^{-1}$, where $q=(4 \pi / \lambda) \sin \left(\frac{\theta}{2}\right)$, with $\theta$ being the scattering angle with respect to the axis of the x-ray beam and $\lambda$ its wavelength. For the given detection system, the observable nanoparticle sizes ranged from 1.6 to $143 \mathrm{~nm}$. Such a setting is optimized for aluminum oxides with a size from several $\mathrm{nm}$ to tens of $\mathrm{nm}$, as can be estimated from our previous work [13].

The circular scattering patterns recorded on the 2D detector were integrated to yield curves of scattered intensity $I$ in $\mathrm{cm}^{-1}$ as a function of $q$. The background scattering intensity was first recorded without laser firing. Then, five measurements were performed in sequence
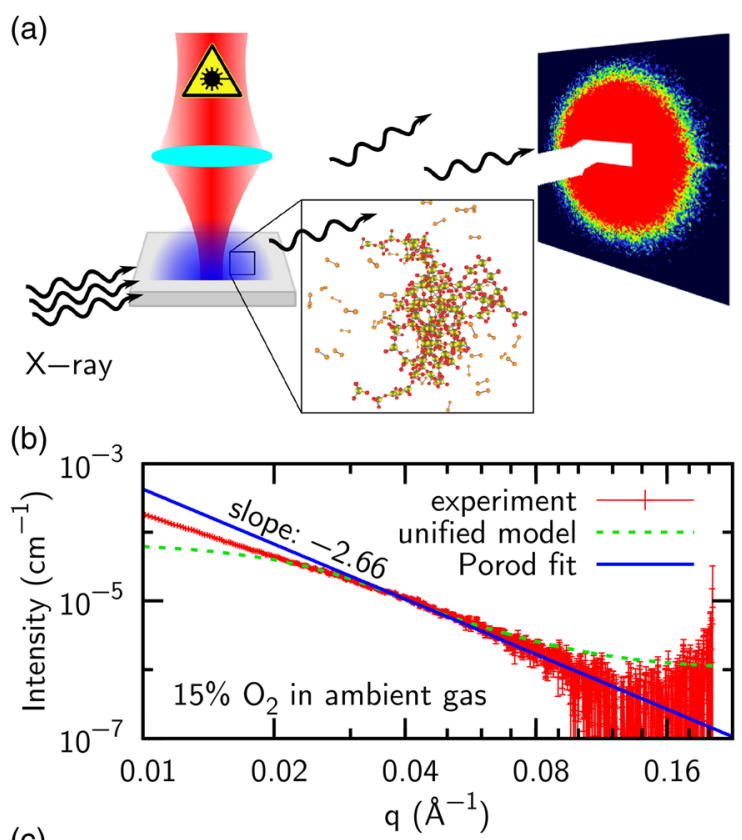

(c)

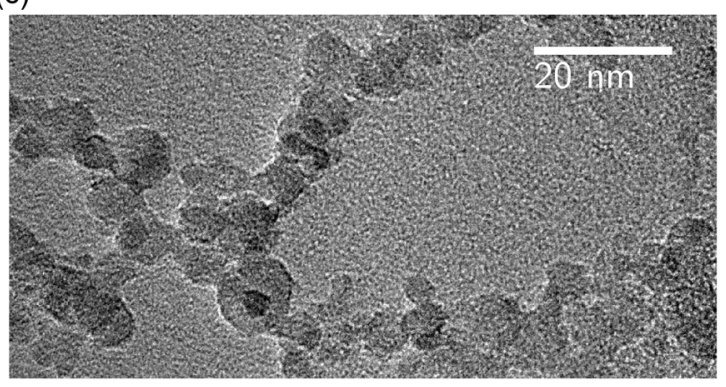

FIG. 1 (color online). (a) Schematic of the experimental setup showing the aluminum target plate (in gray), the ablation laser beam (in red), the ablation plume (in blue), incident and scattered $\mathrm{x}$ rays, a typical aluminum oxide nanoparticle (from the simulation), and a scattering pattern. (b) Typical x-ray scattering intensity $I(q)$ on a log-log scale (limited to the range of $q$ from 0.01 to $0.20 \AA^{-1}$ ), as obtained for nanoparticles produced in an ambient gas with $15 \%$ oxygen (in red), and its fits based on the unified scattering function (dotted green line) and the Porod law near $q=0.1 \AA^{-1}$ (blue line) with a slope of -2.66 . (c) TEM picture of nanoparticles collected by deposition during the $\mathrm{x}$-ray scattering experiment in an ambient gas with $20 \%$ oxygen.

without interrupting the laser, once a permanent plume was established several seconds after the beginning of the laser firing. An acquisition time of $1 \mathrm{~s}$ was necessary to obtain sufficient signal intensity. Figure 1(b) shows the measured scattered x-ray intensity $I$ as a function of $q$ with particles produced within a mixture of $15 \% \mathrm{O}_{2}$ and $85 \% \mathrm{~N}_{2}$ $\left(c_{\mathrm{O}}=15 \%\right)$ in the ambient gas. The experimental intensity $I(q)$ can be fitted using a single level unified scattering function [15] which contains two terms corresponding to the Guinier function [16], and the Porod law that is a power law with an exponent of $-d$, where $d$ is called the Porod slope. The first term is determined by the geometrical parameters of the particles such as their gyration radius $R_{g}$ 


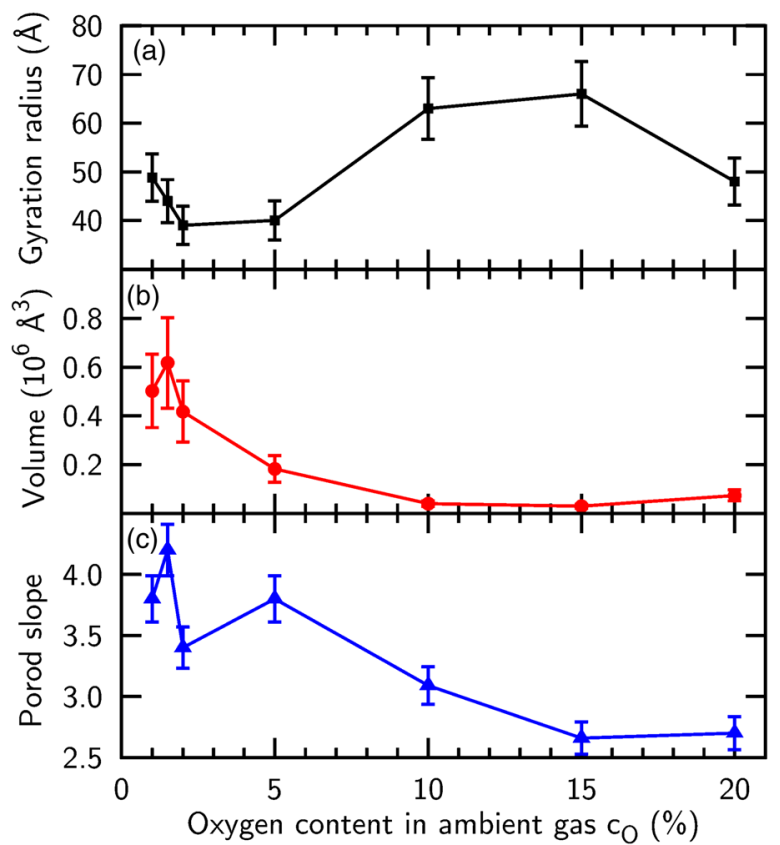

FIG. 2 (color online). Average gyration radius (a), volume (b), and Porod slope (c) of nanoparticles produced in the ablation plume as a function of oxygen content in the ambient gas.

and volume $V$, while the second term reflects the surface structure of the particles. For compact objects with a sharp interface, the Porod slope is $d=4$. In our experiment, the fitting was performed using the IRENA macros package developed by Ilavsky et al. [17]. The obtained results are illustrated in Fig. 1(b), together with the unified fit and the power-law decay fit, resulting in this case in a value of the Porod slope close to $d=2.7$ that indicates a significant degree of roughness. (See the Supplemental Material for more details about the experimental data fitting [18]). The nanoparticles produced were collected during the x-ray scattering experiment with an ambient gas of $20 \%$ oxygen content. TEM observations confirmed the existence of nanoparticles with sizes compatible with the x-ray measurements, even though the particles had further agglomerated into large chains, as shown in Fig. 1(c).

The morphological parameters extracted from the scattered $\mathrm{x}$-ray intensities measured for different oxygen contents in the ambient gas are shown in Fig. 2. The average gyration radius $R_{g}$, the volume $V$ of the nanoparticles thus produced, and the Porod slope $d$ are, respectively, shown as a function of the oxygen content $c_{\mathrm{O}}$ in Figs. 2(a), (b) and (c). As $c_{\mathrm{O}}$ increases, $R_{g}$ first slightly decreases, and then increases to greater values before decreasing again. Minimal values of the gyration radius $R_{g}$ can be found for oxygen contents between $2 \%$ and $5 \%$. In contrast, the volume $V$ exhibits a rather monotonic decrease with increasing $c_{\mathrm{O}}$ except for a minor fluctuation at $c_{\mathrm{O}}=1.5 \%$. The Porod slope has a measured value close to 4 at low oxygen content, clearly indicating sharp interfaces. As the amount of oxygen in the gas increases, this quantity exhibits a sharp decrease above
$1.5 \%$ of oxygen followed by a more gentle increase until the concentration reaches $5 \%$, before globally decreasing toward values as low as 2.7 as the oxygen concentration approaches that of atmospheric air. Those variations in the $1.5 \%-5 \%$ range remarkably correlate with the region where the gyration radius is minimal. Therefore, the present SAXS measurements clearly demonstrate the effects of an increasing oxygen content in the ambient gas from $0 \%$ to $20 \%$ on the overall morphology of the nanoparticles, which so far can be summarized as (i) a decrease in the average volume of the produced nanoparticles, (ii) an evolution of the surface structure of the nanoparticles globally from a sharp interface to one that is rough, and (iii) the existence of an intermediate range of oxygen content, between $2 \%$ and $5 \%$, where the particles again exhibit sharp surfaces as in pure nitrogen, but with a smaller gyration radius.

To interpret the experimental results presented above at the atomistic level, the annealing process of aluminum nanoparticles in ambient gas containing oxygen at various quantities was modeled using the Streitz-Mintmire (SM) reactive potential [19], which stands as one of the few available models for aluminum oxides [20-23] and is expected to provide a good description of a large variety of annealing products. The SM model performs satisfactorily for small Al-O clusters with varying size and stoichiometry when compared with recent electronic structure calculations [24]. The computational protocol relies on molecular dynamics (MD) simulations initiated by randomly placing fixed numbers of $\mathrm{Al}$ and $\mathrm{O}$ atoms, $N_{\mathrm{Al}}$ and $N_{\mathrm{O}}$, respectively, in a soft spherical container, the stoichiometry being determined by the oxygen-aluminum ratio $x_{\mathrm{O} / \mathrm{Al}}=N_{\mathrm{O}} / N_{\mathrm{Al}}$, under the constraint of a total number of atoms $N=N_{\mathrm{Al}}+N_{\mathrm{O}}=2000$. This initial vapor was thermalized at $4000 \mathrm{~K}$ and cooled down in a stepwise way, the temperature being decreased by a constant factor so that it reached $300 \mathrm{~K}$ after 9 steps. Temperature was imposed on the system by a standard Nosé-Hoover thermostat, and at each temperature the MD trajectories were propagated for $50 \mathrm{ps}$ with a 0.5 fs time step, only the last 30 ps being kept for calculating physical properties and the first 20 ps only serving as equilibration. The oxygenaluminum ratio was varied from $x_{\mathrm{O} / \mathrm{Al}}=0$ to $x_{\mathrm{O} / \mathrm{Al}} \approx 10$, and for each value of $x_{\mathrm{O} / \mathrm{Al}}$ the simulation time was thus $450 \mathrm{ps}$, which would have hardly been practical with electronic structure calculations.

Figure 3 shows several properties of the simulated nanoparticles with the structures obtained at the end of the annealing simulation and as a function of increasing oxygen content [Figs. 3(a)-3(b)]. Additional calculations for 500 atoms show only marginal size effects on those properties (see the Supplemental Material [18]), indicating that our computational protocol is robust. Snapshots of those structures at selected oxygen contents are also depicted [Figs. 3(c)-3(g)]. More precisely, we have evaluated the extent of the metallic part within a nanoparticle by 
(a)

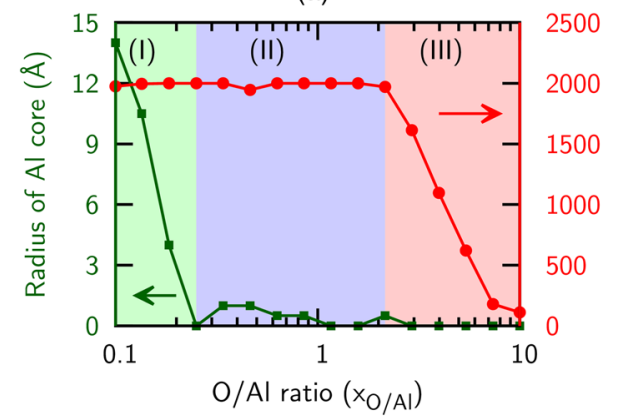

(b)

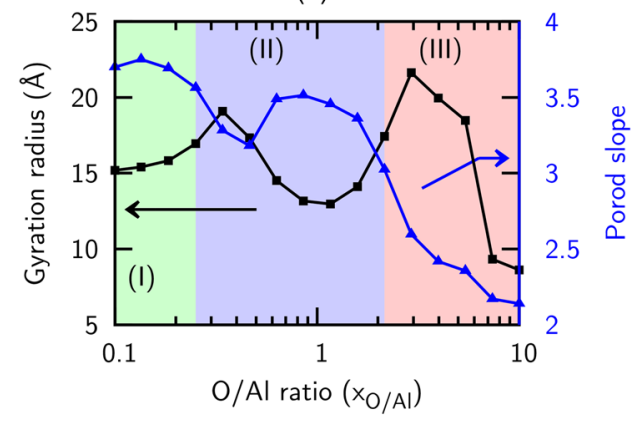

(c) $x_{\mathrm{O} / \mathrm{Al}}=0.00$

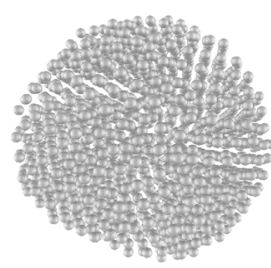

(d) $\mathrm{X}_{\mathrm{O} / \mathrm{Al}}=0.10$

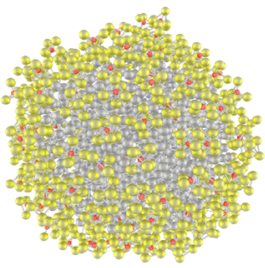

(e) $\times_{\mathrm{O} / \mathrm{Al}}=0.34$

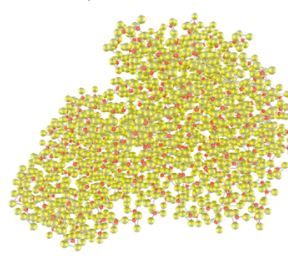

(f) $\mathrm{x}_{\mathrm{O} / \mathrm{Al}}=1.17$

(g) $\times_{\mathrm{O} / \mathrm{Al}}=2.93$

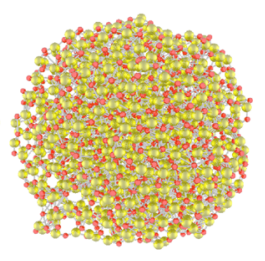

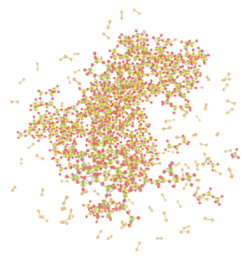

FIG. 3 (color online). Influence of the oxygen content in the ambient gas on the morphological properties of simulated aluminum oxide nanoparticles formed under an oxygen atmosphere. (a) Average radius of the pure aluminum core and total number of connected atoms ( $\mathrm{Al}$ and $\mathrm{O}$ ) in the nanoparticles. (b) Average gyration radius of the nanoparticles and calculated Porod slope. The green, blue, and pink background regions in (a) and (b) indicate the different ranges of the oxygen-aluminum ratio, where the nanoparticles contain a metallic core, are fully oxidized, or are surrounded by residual gas, respectively. Panels (c) to (g) depict typical structures obtained at $300 \mathrm{~K}$ for simulations with different oxygen ratios $x_{\mathrm{O} / \mathrm{Al}}$. The colors of the atoms in (c) through $(\mathrm{g})$ have the following correspondences: gray for metallic Al, red for oxygen, and yellow for oxidized Al.

counting the number of aluminum atoms only bound to other metal atoms and not to any oxygen atom using a simple distance criterion of twice the corresponding diatomic bond length at equilibrium as the cutoff distance (4.39, 3.97, and $3.16 \AA$ for Al-Al, Al-O, and O-O bonds, respectively). The total number of atoms $(\mathrm{Al}+\mathrm{O})$ that constitute the condensed nanoparticle was evaluated by identifying the largest percolated fragment, an atom being considered as belonging to a given fragment if it is connected to other atoms from this fragment according to the above-defined distance criterion. The gyration radius was calculated for those connected nanoparticles, thus avoiding the possible contribution of the residual gas. Finally, the surface roughness of the simulated nanoparticles was determined as the Porod slope, which best fitted the corresponding calculated scattered intensity [25] in the region of $0.05 \AA^{-1}<q<1 \AA^{-1}$, which is relevant for the present nanometer-sized clusters and corresponds quite well to the experimental detection range (see the Supplemental Material [18] for more details about the theoretical Porod slope calculation).

In the absence of oxygen, 2000 aluminum atoms spontaneously form polycrystalline nanoparticles with few stacking faults [Fig. 3(c)]. For $N_{\mathrm{Al}}=500$ the nanoparticles are icosahedral, which is the expected equilibrium shape for the SM potential that essentially reduces to a standard embedded-atom model for a pure metal [19]. It is remarkable that our modeling, spanning much shorter time scales than the actual experiment, is able to produce such highly ordered nanoparticles. We next consider the effects of a moderate amount of oxygen added to the initial aluminum gas. Upon cooling, the gas entirely condenses into a phase of metal nanoparticles surrounded by an oxide shell [Fig. 3(d)], whose thickness increases at the expense of the metal as $x_{\mathrm{O} / \mathrm{Al}}$ increases. According to Fig. 3(a), complete oxidation is reached at an oxygen-aluminum ratio as low as 0.25 . In this regime [zone I in Figs. 3(a)-(b)], the nanoparticles remain essentially spherical, compact and with a smooth surface as inferred from the high Porod slope close to 4 [Fig. 3(b)].

With higher amounts of oxygen (regime II), all available atoms still condense into the nanoparticle but the annealed structure is fully oxidized and increasingly deformed [Fig. 3(e)]. A decreasing Porod slope together with an increasing gyration radius for $x_{\mathrm{O} / \mathrm{Al}}<0.4$ correlate here with a more irregular shape. However, close to the 1:1 stoichiometry, the relatively stable $\mathrm{AlO}$ oxide is preferentially obtained [Fig. 3(f)] with a more pronounced local chemical ordering best seen with the simulation for $N=500$ (see the Supplemental Material [18]), associated with more compact shapes, thus smaller gyration radii and higher Porod slopes. At further increased oxygen content (regime III), the nanoparticles can no longer absorb all available oxygen and become surrounded by residual $\mathrm{O}_{2}$, as illustrated in Fig. 3(g). Restricting the structural analysis to the dominant (largest connected) fragment, the nanoparticles are found to be increasingly disordered and even 
ramified, the residual gas hindering complete condensation. The ramified character of the nanoparticles is associated with an increase in both gyration radius and surface roughness, thus corresponding to a decrease in the Porod slope. As the oxygen content further increases beyond $x_{\mathrm{O} / \mathrm{Al}}>2$, aluminum atoms can no longer bind all available oxygen atoms and the oxide nanoparticle shrinks while keeping a highly ramified structure.

The experimental measurements can now be discussed in the light of the above computational results. First, the observed decrease of the average particle volume as the oxygen content increases can be understood by a decrease of the total number of atoms contained in the major nanoparticle fragment, because of the saturation of the oxidation process. An excess of oxygen with respect to aluminum can stop the nucleation process at an early stage, leaving small free particles in the cloud. Concerning the particle surface structure, it is quite clear that at very low oxygen content, the observed large Porod slope and relatively reduced gyration radius both correspond to compact metallic nanoparticles with a smooth surface. As the oxygen content is increased, the outer layer of the particles is first oxidized. However, at higher oxygen content the particle surface becomes more rugged, which explains the observed dip in the Porod slope as soon as some oxygen is introduced into the system. At specific stoichiometries such as $x_{\mathrm{O} / \mathrm{Al}}=1$, stable aluminum oxides can be obtained, as depicted by Fig. 3(f). Such particular stoichiometries could explain the experimentally observed local maximum in the Porod slope and the local minimum in the gyration radius at $c_{\mathrm{O}}=5 \%$. As the oxygen content further increases beyond 5\%, the decrease of the Porod slope and the increase of the gyration radius observed in the experiment both correspond well to the disordered and ramified surface structures predicted by the simulation. Even though a detailed quantitative comparison is not possible because there is no directly available relation between the oxygen content in the ambient gas $c_{\mathrm{O}}$ and the oxygen-aluminum ratio $x_{\mathrm{O} / \mathrm{Al}}$ used in the simulation, the overall agreement between experiment and simulation is striking. This indicates that we have indeed succeeded in describing the structural features of the nanoparticles formed in the ablation plume using atomistic simulation.

In conclusion, the use of an intense x-ray beam delivered by a synchrotron has allowed in situ characterization of nanoparticles produced in an ablation plume induced by an infrared ns laser pulse on the surface of an aluminum target. Morphological parameters such as the gyration radius, volume, and surface structure of the generated particles could be inferred from SAXS measurements as a function of the oxygen content in the ambient gas. A remarkable finding is the effect of oxidation on the surface structure of the nanoparticles, which become increasingly rough with increasing oxygen content. All observed trends were correctly reproduced by the dedicated MD simulation of nanoparticle formation using a reactive potential. The complementary information obtained at the atomistic level sheds light on the different nanoparticle morphologies ranging from a metallic core-oxidized shell with a smooth surface at low oxygen content, to being fully oxidized with a much more amorphous and ramified surface in the oxygen saturated regime. The good agreement between in situ observations and the modeling based on reactive molecular dynamics highlights the importance of the chemical reactions for the determination of the morphology of the nanoparticles produced in ablation plumes, a key element that is absent in the traditional macroscopic models usually employed to address PLD processes. Future work could be devoted to extending this methodology to other oxides of application relevance such as titanium or silver.

The authors would like to acknowledge the financial and scientific support of Synchrotron SOLEIL and in particular Dr. Javier Perez and his staff on the SWING beamline. The authors gratefully acknowledge the support from the Pôle Scientifique de Modélisation Numérique (PSMN) at ENS Lyon for a generous allocation of computer resources.

*Corresponding author.

jin.yu@univ-lyon1.fr

Corresponding author.

brian.mitchell@univ-rennes1.fr

[1] D. H. Lowndes, D. B. Geohegan, A. A. Puretzky, D. P. Norton, and C.M. Rouleau, Synthesis of novel thin-film materials by pulsed laser deposition, Science 273, 898 (1996).

[2] P. R. Willmott and J. R. Huber, Pulsed laser vaporization and deposition, Rev. Mod. Phys. 72, 315 (2000).

[3] D. Dijkkamp, T. Venkatesan, X. D. Wu, S. A. Shaheen, N. Jisrawi, Y. H. Min-Lee, W. L. McLean, and M. Croft, Preparation of $\mathrm{Y}-\mathrm{Ba}-\mathrm{Cu}$ oxide superconductor thin films using pulsed laser evaporation from high $T_{c}$ bulk material, Appl. Phys. Lett. 51, 619 (1987).

[4] K. Ramamoorthy, C. Sanjeeviraja, M. Jayachandran, K. Sankaranarayanan, P. Misra, and L. M. Kukreja, Development of a novel high optical quality $\mathrm{ZnO}$ thin films by PLD for III-V opto-electronic devices, Curr. Appl. Phys. 6, 103 (2006).

[5] A. V. Kabashin, J.-P. Sylvestre, S. Patskovsky, and M. Meunier, Correlation between photoluminescence properties and morphology of laser-ablated $\mathrm{Si} / \mathrm{SiOx}$ nanostructured films, J. Appl. Phys. 91, 3248 (2002).

[6] Y. Nakata, J. Muramoto, T. Okada, and M. Maeda, Particle dynamics during nanoparticle synthesis by laser ablation in a background gas, J. Appl. Phys. 91, 1640 (2002).

[7] I. A. Movtchan, W. Marine, R. W. Dreyfus, H. C. Le, M. Sentis, and M. Autric, Optical spectroscopy of emission from $\mathrm{Si}-\mathrm{SiOx}$ nanoclusters formed by laser ablation, Appl. Surf. Sci. 96-98, 251 (1996).

[8] D. B. Geohegan, A. A. Puretzky, G. Duscher, and S. J. Pennycook, Time-resolved imaging of gas phase nanoparticle synthesis by laser ablation, Appl. Phys. Lett. 72, 2987 (1998). 
[9] R. F. Wood, K. R. Chen, J. N. Leboeuf, A. A. Puretzky, and D. B. Geohegan, Dynamics of Plume Propagation and Splitting during Pulsed-Laser Ablation, Phys. Rev. Lett. 79, 1571 (1997).

[10] Z. Chen and A. Bogaerts, Laser ablation of $\mathrm{Cu}$ and plume expansion into $1 \mathrm{~atm}$ ambient gas, J. Appl. Phys. 97, 063305 (2005).

[11] T. E. Itina, A. A. Katassonov, W. Marine, and M. Autric, Numerical study of the role of a background gas and system geometry in pulsed laser deposition, J. Appl. Phys. 83, 6050 (1998).

[12] S. di Stasio, J. B. A. Mitchell, J. L. LeGarrec, L. Biennier, and M. Wulff, Synchrotron SAXS in situ identification of three different size modes for soot nanoparticles in a diffusion flame, Carbon 44, 1267 (2006).

[13] L. Lavisse, J.-L. Le Garrec, L. Hallo, J.-M. Jouvard, S. Carles, J. Perez, J. B. A. Mitchell, J. Decloux, M. Girault, V. Potin, H. Andrzejewski, M. C. Marco de Lucas, and S. Bourgeois, In-situ small-angle x-ray scattering study of nanoparticles in the plasma plume induced by pulsed laser irradiation of metallic targets, Appl. Phys. Lett. 100, 164103 (2012).

[14] http://www.synchrotron-soleil.fr/Recherche/LignesLumiere/ SWING.

[15] G. Beaucage, Approximations leading to a unified exponential/power-law approach to small angle scattering, J. Appl. Crystallogr. 28, 717 (1995).

[16] A. Guinier and G. Fournet, Small-Angle Scattering of $X$-Rays, Structure of matter series edited by M. G. Mayer (Wiley, New York, 1955).
[17] J. Ilavsky and P. R. Jemian, Irena: Tool suite for modeling and analysis of small-angle scattering, J. Appl. Crystallogr. 42, 347 (2009).

[18] See Supplemental Material at http://link.aps.org/ supplemental/10.1103/PhysRevLett.115.246101 for more detailed information about the determinations of the experimental and theoretical Porod slopes, as well as the results of an additional simulation with 500 atoms.

[19] F. H. Streitz and J. W. Mintmire, Electrostatic potentials for metal-oxide surfaces and interfaces, Phys. Rev. B 50, 11996 (1994).

[20] P. Vashishta, R. K. Kalia, A. Nakano, and J. P. Rino, Interaction potentials for alumina and molecular dynamics simulations of amorphous and liquid alumina, J. Appl. Phys. 103, 083504 (2008).

[21] I. Lazić and B. J. Thijsse, An improved molecular dynamics potential for the Al-O system, Comput. Mater. Sci. 53, 483 (2012).

[22] X. W. Zhou, H. N. G. Wadley, J.-S. Filhol, and M. N. Neurock, Modified charge transfer - embedded atom method potential for metal/metal oxide systems, Phys. Rev. B 69, 035402 (2004).

[23] T. Campbell, R. K. Kalia, A. Nakano, P. Vashishta, S. Ogata, and S. Rodgers, Dynamics of Oxidation of Aluminum Nanoclusters using Variable Charge Molecular-Dynamics Simulations on Parallel Computers, Phys. Rev. Lett. 82, 4866 (1999).

[24] G. D. Förster, F. Calvo, and F. Rabilloud (unpublished).

[25] C. M. Sorensen, Light scattering by fractal aggregates: a review, Aerosol Sci. Technol. 35, 648 (2001). 